\title{
Die Formen des Genitiv II Pluralis im Alten Kalevala
}

Die Lieder in der ersten - 1835 erschienenen - Ausgabe des Kalevala stammen meist aus solchen Gebieten, wo der Genitiv I Pl. verwendet wird. Im Karelien jenseits der Ostgrenze ist der Genitiv I ausschliesslich, wie auch im grössten Teil von Finnisch-Karelien. Der Genitiv II Pl. wird nur in der Gegend von Nurmes benutzt; hierin schliesst sich der Dialekt wie auch in einigen anderen Punkten den Mundarten an, die in Kainuu, der nördlichen Nachbarprovinz, gesprochen werden. ${ }^{1}$ Im folgenden soll die Frage beantwortet werden, welche der im Alten Kalevala auftretenden Formen des Genitiv II Pl. auf der Volksdichtung beruhen, welche dagegen auf Lönnrot zurückgehen. Als Quelle wurde Väinö Kaukonens Arbeit »Vanhan Kalevalan kokoonpano» (Die Zusammensetzung des Alten Kalevala) (Teil I 1939, Teil II 1945) benutzt. Um Fehler zu vermeiden, die aus Versehen bei dem Vergleich von Zeilen haben entstehen können, sind die Stellen an der gedruckten Ausgabe der Suomen Kansan Vanhat Runot überprüft worden.

Sprachlich gesehen haben die von Lönnrot hinzugefügten Formen des Genitiv II Pl. insofern Bedeutung, als dadurch mehr westliche Elemente in das Epos gelangten als die zugrundeliegenden Volkslieder voraussetzen würden. Das eigentliche Problem bilden jedoch die Fälle, die zusammenhängen mit den im Verwendungsgebiet von Genitiv I aufgezeichneten Liedern und auf diesem Wege ins Kalevala gelangt sind.

Aufgrund der heutigen Kenntnisse scheinen die folgenden Zeilen mit einem Gen. II Pl. Lönnrots Handschrift zu sein: ${ }^{2}$

\footnotetext{
${ }^{1}$ Lauri Kettunen, Suomen murteet III B, Karte 186.

${ }^{2}$ Die Verse sind alphabetisch nach dem Wort geordnet, das im Gen. II Pl. steht. Die Formen sind hier kursiviert. Die Nummern nach den
} 
Pahin astiain pesiä $(17,538)$ - Gebildet aus dem Vers Aina astian pesiä $\left(\mathrm{I}_{2} 793,20\right)$

Apu einetten emistä $(4,353)$ - Apu einisten (drüber: eineisten) emistä ( I $_{4}$ 168, 186). Das einetten im Alten Kalevala ist formal richtig, doch ist der Vers bedeutungsmässig nicht zu verstehen. Allerdings ist auch der ursprüngliche Vers dunkel. Es handelt sich offenbar um ein verderbtes Wort; der Vers ist denn auch in der neuen Ausgabe des Kalevala weggelassen worden. Auf eine Verballhornung weist auch der vorangehende Vers im Gesang hin: J[uma]loista tunti tullo (im AK Jumaloista turva tulko).

Leppäharjuin hartioitse $(11,263)$ - Leppäharjun harteitse (I1 488, 120)

Nyt sie juokse Hiitten hirvi $(7,46)-$ H[iien] h[irvi] (I2 867, 27)

Kivutar kipuen eukko $(25,179)$ - »Von Lönnrot hinzugefügt.» (Kaukonen)

Kuukauet kujaisten suussa $(28,182)$ - Der Vers hat bei Kaukonen keinen Kommentar. Das mögliche Original ist also unbekannt.

Kuningasten kunnioiksi $(22,366)$ - Wie der vorangehende Vers.

Läksi lammasten keralla $(32,36)$ - "Von Lönnrot gebildet.» (Kaukonen)

Lapin lasten tanterille $(6,6$ und 7, 50) - In dem mit ersterem zusammengestellten Vers "Lapin talvi tanterille» fehlt lasten, in dem mit letzterem zusammengestellten steht ein anderes Wort: Lapin lastu tanterille ( $\left.\mathrm{I}_{2} 867,30\right)$. Lönnrot hat also die Veränderung lastu $\rightarrow$ lasten vorgenommen. | Ahin lasten aartehiksi $(23,405)$ - Kaukonen: Kein Kommentar.

Läpi liikkuvain lihojen $(25,16)$ - Läpi liikkuvan lihan (XII2 $6709,210)$

Päät menevi kauppamiesten $(1,269)$ - Päät menee kaupa-

Versen verweisen auf das Alte Kalevala (z.B. 17,538=17. Gesang, Zeile 538) und auf die im Sammelwerk Suomen Kansan Vanhat Runot veröffentlichten Lieder (z.B. I2 793, $20=$ Teil I, Band 2, Lied 793, Vers 20). 
miehen (I1 79a, 29) | Miesten syöjälle selälle $(6,237)$ - Miehen s. S. (VI1 4, 51) | Miesten syöjästä kylästä $(11,5)$ - Miehen syöjästä kyläst[ä] (I1 469, 6) | Miesten joukko juominkihin $(13,380)$ - "Von Lönnrot gebildet.» (Kaukonen) | Sammosta sanomat miesten $(23,10)$ - »Von Lönnrot gebildet.» (Kaukonen)

Lasten laulut, naisten naurut $(30,109)$ - "Von Lönnrot gebildet.» (Kaukonen)

Katson päälle orittesi $(30,182)$ - "Von Lönnrot gebildet." (K:nen)

Otavaisten olkapäitse $(5,129)$ - Otav[aisen] 0. (I4 168, 177) Pohjan poikajen tiloilla $(2,134)$ - "Der Vers ist von Lönnrot gebildet.» (K:nen) | Pohjan poikajen tulille (6,5 und 26) "Von L. gebildet.» (K:nen) | Ole puolla poikajesi $(24,281)$ Ota puolla poikaisi (XII2 7863,49$)$ Spr. Kajaani (Lnr.). Die Form poikaisi ist ebenfalls ein Gen. II Pl., doch hat Lönnrot sie lautlich archaischer gestaltet.

Päitä suonten solmieli (4,307 und 327) - Der Vers ist in beiden Zusammenhängen von L. gebildet, doch begegnet die Form suonten auch in einigen auf der Volksdichtung beruhenden Versen, wie weiter unten zu sehen sein wird.

Näkyville Pohjan portin, Rautausten ulvomille $(17,154)$ »Offensichtlich von L. gebildet.» (K:nen)

Urosten upottajalle $(6,238)$ - Urohon u. (VI1 4, 51)

Vallatonten vaattehiksi $(28,279)$ - Valtamiehen voatteiksi ( $\mathrm{I}_{4}$ 1251, 46)

Töille virtten työntelihen $(16,178)$ - »Von L. eingefügt.» (K:nen). Der Vers besitzt eine genaue Entsprechung auch an einer anderen Stelle $(14,217)$, wo er auf ein Volkslied aufbaut.

Die in den folgenden Versen des Alten Kalevala auftretenden Formen des Gen. II Pl. stammen aus der Volksdichtung. In den alten Sammlungen sind die Angaben über Sänger und Aufzeichnungsort jedoch oft lückenhaft, so dass man vielleicht nur den Sprengel kennt, in dem das Lied aufgezeichnet ist (z.B. Spr. Kajaani), mitunter steht auch dort noch ein Fragezeichen (z.B. Geg. Kemi?). Die Sprengel von Kajaani und Kemi gehören zu dem Gebiet, wo der Gen. II Pl. von alters her volkstümlich ist, so dass man erwarten kann, dass diese Formen auch in den Liedern erscheinen, die dort aufgezeichnet sind. Hin- 
sichtlich der Belege in Finnisch- und Russisch-Karelien ist die Angelegenheit nicht so klar, da die Formen zumindest nicht auf der heutigen gesprochenen Sprache beruhen.

Hammasten hajoamatta $(14,337)$ - Hammasten hajoamata (XII1 341, 21 Geg. Kemi?). Der Abessiv hajoamata entspricht nicht dem Dialekt von Kemi, wohl aber dem von Kainuu. Hammasten hajottamasta $(19,255)-$ H. h. (XII2 6481, 67 Suomussalmi) | Kova hammasten kolina $(28,498)$ - »Vermutlich aus einer verlorengegangenen Variante.»(K:nen). Ein gleichlautender Vers ist in einem Lied enthalten, das Kaarle Krohn in Ristijärvi i.J. 1882 aufgezeichnet hat.

Kapustain kaavistaja $(17,540)$ - Kapustaj[e]n k. (I2 758, 209, Latvajärvi)

Kitahan kirokavetten $(6,214)$ - Kitah[an] kiro kavetten (I2 758, 156, Latvajärvi | Ders. Vers (17, 225 und 474) Kitaan k. und Kitah[an] k. (I2 759, 75 und 92, Latvajärvi)

Lummetkoirain koista $(10,157)$ - lumme koirain kotoa (VII 4 1594, 44, Pielisjärvi). Der Gen. II Pl. ist auch in der Mundart von Pielisjärvi volkstümlich; in einer entsprechenden Dialektprobe in Suomen Kansan Murrekirja Teil II finden sich z.B. die Formen pitäjäläesten, lasten und miesten.

Aina lastesi apuna $(24,282)$ - Ders. Vers. (XII2 7863, 50 Spr. Kajaani) | Ei oo laulut lasten laulut, Lasten laulut naisten naurut (30, 108 und 109) - Ersterer Vers derselbe (XII2 323, 1, [Spr. Kajaani]), letzterer von Lönnrot gebildet (K:nen).

Luisten lukkojen sisälle (14, 332) - Luisten lukkujen s. (XII1 341, 18, [Geg. Kemi?]) | Läpi luisten leukaluijen $(19,361)$ - L. 1. leukaluihen (VII5 3848, 13, Eno) | Alla luisten polviensa, Rautasten rusamiensa (28, 511 und 512) - A. 1. polviinsa, R. rusamisinsa (XII2 6827, 339 und 40, Sotkamo)

Lusikkain luutustaja $(17,539)$ - Ders. Vers (I2 758, 208, Latvajärvi)

Miesten syöjähän siahan $(2,35)$ - Miest[en] syöj[ähän] kyl[ähän] (I1 54, 147, Latvajärvi) | Muien miesten metsimaille (7, 146) - Muihen miesten mehtimaille (VII5 3295, 5, Mlomantsi) | Eipä miesten muienkana $(7,279)$ - Eipä miesten muitenkaan (VII5 3359, 144, Kesälahti) | Tietomiesten tienohilta $(10,153)$ - tieto miesten tienohille (VII 4 1594, 38, Pielis- 
järvi) | Mäkipäistä möyrymiesten (10, 156) - M. mäyrymiesten (VII4 1594, 41, Pielisjärvi) | Miesten seurojen sekahan (17, 296) - Miesten seuroin sekoon (I2 774, 289, Tsena) | Miesten mielijuohtehista $(24,277)$ - "Aus einem Zauberspruch von L. übernommen.» (VII 4 3062, 21, Nurmes) | Miesten verroille veteä $(6,46)$ - Ders. Vers ( $\mathrm{I}_{4} 410,92$, Latvajärvi) | Miesten miekan tutkamia $(10,53)$ - Miesten miekkojen teriä ( $\mathrm{I}_{1} 403$, 49, Tsena)

Naisten neulojen neniä $(10,18$ und 10,51$)$ - Ders. Vers (I1 420, 26, Lonkka und I1 403, 47, Tsena)

Mieli muien morsianten $(15,140)$ - mitä muihen m. (VII2 2064, 2, Ilomantsi)

Otavaisten olkapäille $(30,136)$ - Der Vers begegnet in den Liedern der sog. Q-Sammlung (n:0 338, 4), die Lönnrot in Kerimäki oder Ilomantsi aufgezeichnet hat (Kaukonen, Vanhan Kalevalan kokoonpano I, S. 505).

Pieni peitetten sisässä $(15,268)$ - Ders. Vers (VII3 4456, 2, Nurmes)

Rautasten rusamiensa $(28,512)-$ R. rusamisinsa (XII2 6827, 40, Sotkamo)

Läpi suonten soljuvitten $(25,16)$ - L. s. soljuitten (XII2 6709, 211, Suomussalmi). Die Form wirkt befremdlich sowohl in ihrer ursprünglichen Aufzeichnung als auch in der von Lönnrot korrigierten Fassung. Erwartungsgemäss wären soljuvien und soljuvain, wobei erstere auch den Anforderungen des Versmasses genügen würde. Die Form soljuitten passt nur so gesprochen (gesungen) zum Versmass, dass zwischen $u$ und $i$ die Silbengrenze ist. Soljuitten würde den Nom.Pl. soljuet voraussetzen, wofür jedoch keine Belege vorliegen. Das soljuvitten im AK ist eine Verbform, deren Stamm soljua lautet. Auf demselben Wort basiert auch soljuvaisten im Neuen Kalevala. In Lönnrots Wörterbuch steht s.v. soljuva als Beispiel der genannte Vers des AK, bei Ganander jedoch suonia solisowia (nicht soljuvia).

Soria on suonten vaimo $(4,310)$ - sorree on suonten vaimo (VII3 907, 1, Nurmes) | Soma suonten ketreäjä (4, 312) - s. s. keträäjä (VIIз 907, 3, Nurmes)

Urosten upottajahan $(2,36)$ - Urosten upott[ajaan] (I1 30, 61, Akonlaksi) | Urosten eräsaloille $(7,147)$ - U. eräsalolle 
(VII5 3295, 6, Ilomantsi) | Sueksen urosten sulho $(17,32)-$ Sueksii u. s. (I1 95, 192, Lonkka) | Urosten upottajille $(5,81)$ Entsprechung fehlt; hingewiesen worden ist auf das Urosten upottajaan im obigen Vers, das also als Muster hat dienen können.

Töille virtten työntelihen $(14,217)-$ v[irtten] työntelih[en] (I1 362, 23, Latvajärvi). Derselbe Vers steht auch an einer anderen Stelle $(16,178)$, wo er nach Kaukonen durch Lönnrot hingelangte.

Wie aus den obigen Ausführungen hervorgeht, haben zahlreiche Formen des Gen. II Pl., die im Alten Kalevala stehen, in den entsprechenden Volksliedern eine Entsprechung. Die meisten Lieder wurden in Kainuu und in Pielis-Karelien aufgezeichnet, in Gebieten also, wo die genannten Genitive dem Dialekt entsprechen. Formen in Liedern, die südlich dieses Gebietes (in Ilomantsi, Kesälahti, Kerimäki) aufgezeichnet wurden, können nicht als volkstümlich angesehen werden.

Problematischer ist jedoch, dass von diesen Genitivformen mehr als zehn in weissmeerkarelischen Liedern enthalten sind, obwohl der karelische Dialekt jenseits der Grenze sonst den Gen. I Pl. aufweist. Die Formen, die in Teil I der Lieder aus dem Bezirk Weissmeerkarelien auftreten, lassen sich folgendermassen gruppieren.

1. Zweisilbige vokalstämmige Wörter; Belege gibt es wenig: akkain (415, 30), vajajen (328, 79). Borenius, der das Lied aufgezeichnet hat, unterstrich das a der zweiten Silbe des Wortes vajajen, so dass es sich nicht um einen Schreib- oder Druckfehler handelt. Allerdings begegnet auch das dem Karelischen entsprechende vajojen in den Liedern (valvatti vajojen peässä, 120, 24).

2. Zweisilbige, auf Vokal endende Wörter, die einen Vokalund Konsonantstamm haben: hiitten $(1,90 ; 26,88 ; 155,89$; $553,21 ; 617,113 ; 654,48)$, hiiten ( $<$ hiitten) (9a, 250; 145, $156)$, koarten $(495,8)$, lasten $(22,273 ; 36,57 ; 193,42 ; 442,273)$, vuolten $(298 \mathrm{a}, 67)$.

3. Einsilbige, auf $-s$ auslautende Stämme: miesten (9a, 68 und $94 ; 15,144 ; 18,62 ; 42,24 ; 54,147 ; 79,26 ; 88,34 ; 116,40$; 321,$22 ; 322,14 ; 399,33 ; 508,35 ; 520,11$ und $102 ; 522,6 ; 670$, $30 ; 670 \mathrm{a}, 28 ; 694,44)$, naisten $(42,23 ; 58 \mathrm{a}, 107 ; 60,42 ; 124,7$; 
$399,33 ; 456,54)$. Es sind nicht alle Belege aufgezählt worden, weil dieselben Verse ungefähr in der gleichen Form viele Male wiederkehren und auch in Liedern mit verschiedener Thematik (z.B. "miesten miekkojen teriä» und »naisten nieklojen [oder neulojen] neniä»).

4. Zwei- und mehrsilbige, auf $-l,-n,-r$, und $-s$ endende Stämme: hevoisten $(616,2)$, keihästen (83a, 186 und 187), kulokasten $(616,3)$, lammašten $(227,39)$, lämminten $(1,26)$, petäjäisten $(16,31)$, urosten $(9 \mathrm{a}, 69 ; 78,78 ; 329,18 ; 670 \mathrm{a}, 29)$, vaivaisten $(101,58)$, vierasten $(372,69)$.

5 . Ursprünglich auf $-h$ und $-k$ auslautende Stämme: vaatteitten $(448,2)$, vaattehitten $(449,3)$, venetten $(280 \mathrm{a}, 4)$. Das in der Form venetten auftretende $t t$ entspricht nicht der karelischen Sprache, denn in den konsonantstämmigen Formen der auf - $h$ ausgehenden Wörter hat sich $h t$ erhalten (z.B. Partit. venehtä). Andererseits ist das $h$ der Form vaattehitten analog, da das Wort ursprünglich auf $-k$ endet. Ein erwartungsgemässer Gen.Pl. steht z.B. in dem Vers "Läksi voatteijen pesuhun» $(452,2)$.

Bis auf wenige Ausnahmen haben die Sänger die Formen richtig angewendet, und auch lautlich sind die Formen völlig: korrekt. Im folgenden Fall scheint der Sänger den pluralischen Genitiv für singularisch gehalten zu haben: »Köyhän miesten peä mänövi, Rikkahan kalu kuluvi» $(88,34)$, im folgenden wiederum steht der Gen.Pl. in der Funktion des Akk.Sg.: »Joka päivä miesten syö, Parahana kaksi miestä» $(520,11)$. Zur ersten Stelle bemerkt Borenius: "Der Sänger hat die Form miesten als Singular aufgefasst; im karelischen Dialekt immer miehien.»

Ein zuverlässiges Bild über die Verwendung des Gen.Pl. in den heutigen weissmeerkarelischen Dialekten vermittelt die Sprachprobensammlung "Vienan kansa muistelee» von Pertti Virtaranta. Ich habe aus Teil I (S. 1-401) alle Formen exzerpiert; für den Gen. II Pl. blieb das Ergebnis recht dürftig. Solche Formen begegnen nämlich nur in Bruchstücken von Liedern, die das Kalevala-Versmass haben. Es sind so wenig Belege, dass sie hier aufgezählt werden können:

Herheläińi hiittel lintu, meheläińi mettel lintu (S. 131) | Meheläińi meitten lintu, herheläińi hiitten lintu (134). Wenn metten der Gen.Pl. vom Wort mesi ist - was sein kann - dann kann das $i$ der Form meitten aus der Form hiitten in der be- 
nachbarten Zeile stammen. | Eihän täss ole miesten surma, eikä kuoloma urosten (11) | miesten syöjästä kylästä, urosten upottajasta (35).

Vergleichshalber zitiere ich auch Proben von Formen des Gen. I Pl. und bringe sie in derselben Reihenfolge wie oben. Die Zahlen nach den Formen verweisen auf die Seiten im Buch von Virtaranta, $\mathrm{R}$ bezeichnet Text in gebundener Rede, in Versform.

1. aitojem perillä (326), heińien (370), herrojen (328 R), ilmoin (110 R), jalkojev välistä (397), jyviem peälittši (389), kalojej jumala (260), koirien (84), lauttšojen alta (153), lehmiev vasoja (13 R), luhtijen (Nom.Sg. luhta) alla (302), miekkojen (109), opreśoijen eteh (107), pahojej jälkilöiśtä (78), pitkien (89), pohrottšoijev välillä (326), potakkojen kera (226), varassotien aikana (96), kolmien śuutkiem peästä (164), vajojem päiśśä (113 R), vakkojem peällä (272), vanhojen (173), vanhempiel lapsien (250).

2. lapsiel luota (89), nuoriev voatteita (173), pieńiel lintujen (350), soarien (98), varsiem peällä (190), veittšijen kera (382),

3. pahojem miehijen (89), naiśiev viinan (122), naiśienki (370), toiśien keralla (88).

4. hampahiev välih (115 R), jouttšeńiej joruhun (35 R), kintahiem peällä (190), kirvehien (84), pentuśiej jälkilöillä (317), räpähien (35 R), semmośiem mäntyjen (282), tahtahien (286), varpahiev välit (148).

5. liśteijem peällä (Nom.Pl. liśtiet) (222), läksi voatteijem pesulla (35 R), voatteijem peällä (190), venehiev välistä (268), viikattehien kera (81).

6. Als eigene Gruppe sind die Formen der einsilbigen Nomina zu erwähnen. Ein solcher, in den westlichen Dialekten üblicher Typus wie maitten fehlte im Alten Kalevala und auch in den alten Volksliedern. Den finnischen Ostdialekten ist er fremd, desgleichen den weissmeerkarelischen Mundarten. Einige Beispiele aus Virtarantas Veröffentlichung: jäijel lähtieśśä (352), muijen (288), ńiijen kera (30), näijen tyttöjen (299), puijel latvoiśśa (351). Nur in den Formen des Gen.Pl. von Personalpronomina fehlt das $i$ : meän kylässä (16), meän talossa (31). Nach Genetz (TVKK S. 202) entsprechend teän (= teidän) und heän (= heidän). Wie sie auch zu erklären sind: sie bilden 
höchstens die Ausnahme von der Regel, dass im Karelischen der $i$-Charakter zum Gen.Pl. gehört. Die Formen des Gen. II Pl. können somit keine karelischen Eigenheiten sein. Wenn nämlich in der Morphologie des heimatlichen Dialektes eines Sängers ein Element fehlt, das in seinen Liedern erscheint, muss vorausgesetzt werden, dass er diese Verwendung von Sprachträgern einer anderen Mundart erlernt hat. In Frage käme dabei Nordostbottnien, in erster Linie das Gebiet zwischen Raahe und Kemi, wo es bereits im Mittelalter Bewohner gab, die den Häme- und einen auf dem Karelischen basierenden Dialekt sprachen. Vom Frieden zu Pähkinäsaari (Schlüsselburg) (1323) bis zum Frieden von Täyssinä (1595) gehörte dieses Gebiet zum Einflussbereich von Nowgorod, doch gab es dort schon vor dem 17.Jh. auch lutherische Gemeinden (wenigstens Ii, Liminka und Hailuoto). Der Überlieferung zufolge sind gerade die Vorfahren jener Sänger, deren Lieder eine zentrale Stellung im Alten Kalevala einnehmen, aus dem Westen nach Weissmeerkarelien gezogen, aus Nordostbottnien. ${ }^{1}$

Es ist offenbar das Verdienst des Versmasses, dass sich der Gen. II Pl. erhalten hat. Veränderungen wie akkain $\rightarrow$ akkojen, miesten $\rightarrow$ miehien, hammasten $\rightarrow$ hampahien hätten ja die Silbenzahl erhöht und somit das Kalevala-Versmass gestört. In gewissem Masse kam es jedoch zu einer Anpassung an den regionalen Dialekt, wie z.B. aus den folgenden Gegenüberstellungen hervorgeht: ${ }^{2}$ Kullat on lašten kukkasia $(193,42) \rightarrow$ Kullat on lapsien kukkasia (182, 42), Läpi lämminten lihojen $(1,26) \rightarrow$ Läpi lämpymien lihojen $(9,28)$, Naisten nieklojen neniä $(399,33) \rightarrow$ Naisien n. n. $(611,18)$. Wenn die pluralische Form umgeändert wurde in eine singularische, hat sich das Versmass besser erhalten: Miesten miekkojen teriä $(604,23) \rightarrow$ Miehen m.m. (611, 17), Akkain neulojen neniä $(415,30) \rightarrow$ Akan ńeulojen neńiä $(694,40)$. Die Formen des Gen. II Pl. sind jedoch i.a. in den Liedern bewahrt worden, auch wenn sie der karelischen Sprache fremd sind.

Veikko Ruoppila

${ }^{1}$ Ich verweise auf meine Arbeit »Kalevala ja kansankieli» S. 183184, wo die Frage nach der Heimat der Gesänge im Alten Kalevala eingehend behandelt ist.

${ }^{2}$ Die Verse stammen aus Band 1 von Teil I (SKVR I). 